\title{
IMPROVED UPPER BOUNDS FOR THE AVAILABILITIES IN A FIXED TIME INTERVAL FOR MULTISTATE MONOTONE SYSTEMS
}

\author{
BENT NATVIG, ${ }^{*}$ University of Oslo
}

\begin{abstract}
It is here shown how the upper bounds given in Funnemark and Natvig (1985) can easily be improved.

UNAVAILABILITIES: MAINTENANCE：INTERDEPENDENT COMPONENTS
\end{abstract}

In a recent paper, Funnemark and Natvig (1985) derived upper and lower bounds for the availability, $h_{\phi}^{j(I)}$, and unavailability, $g_{\phi}^{j(h)}$, to any level $j$, in a fixed time interval $I$ for multistate monotone systems based on corresponding information on the multistate components. These are assumed to be maintained and interdependent. Such bounds are of great interest when trying to predict the performance process of the system, noting that exact expressions are obtainable only for trivial systems. The bounds given in Funnemark and Natvig (1985) generalize the ones given in Natvig (1980) covering traditional binary theory.

The lower bounds given in these papers are supposed to be good, whereas most upper bounds are good only for short interval lengths. For long interval lengths most upper bounds may be very poor. It is the aim of this short note to indicate how each of these poor upper bounds can easily be improved, simply taking the infimum of all corresponding upper bounds calculated for each fixed point of time in the interval.

Concerning the notation and main concepts in multistate reliability theory we refer to Funnemark and Natvig (1985). If $I=[t, t]$, we replace the $I$ in the notation by $t$. Note that

$$
h_{\phi}^{j(I)}+g_{\phi}^{j(I)} \leqq 1
$$

whereas

$$
h_{\phi}^{j(t)}+g_{\phi}^{j(t)}=1 \quad \forall t \in I .
$$

It is the application of the former relation that causes the poor upper bounds for long intervals $I$.

The bounds for $h_{\phi}^{j(I)}$ and $g_{\phi}^{j(I)}$ in Theorem 3.1 of Funnemark and Natvig (1985) are supposed to be good even for fairly long intervals $I$, but seem of little practical value due to the complexity of the bounds. As an illustration of the technique of improving the very poor upper bounds we present the improved version of Corollary 3.7 of the above-mentioned paper.

Theorem. Let $(C, \phi)$ be a multistate monotone system with the marginal performance processes of its components being independent and each of them associated in $I$.

Received 10 December 1985; revision received 27 February 1986.

* Postal address: Institute of Mathematics, University of Oslo, P.O. Box 1053, Blindern, 0316 Oslo 3, Norway. 
Furthermore for $j \in\{1, \cdots, M\} \quad$ let $y_{k}^{j}=\left(y_{1 k}^{j}, \cdots, y_{n k}^{j}\right), \quad k=1, \cdots, n^{j}\left(z_{k}^{j}=\right.$ $\left.\left(z_{1 k}^{j}, \cdots, z_{n k}^{j}\right), k=1, \cdots, m^{j}\right)$ be its minimal path (cut) vectors to level $j$. Also denote the availability and unavailability to level $j$ in $I$ for the $i$ th component by $p_{i}^{j(I)}$ and $q_{i}^{j(I)}$ respectively and introduce the $n \times M$ matrices

$$
\boldsymbol{P}_{\phi}^{(I)}=\left\{p_{i}^{j(I)}\right\}_{\substack{i=1, \cdots, n \\ j=1, \cdots, M}} \boldsymbol{Q}^{(I)}=\left\{q_{i}^{j(I)}\right\}_{\substack{i=1, \cdots, n \\ j=1, \cdots, M}}
$$

Define

$$
\begin{aligned}
l_{\phi}^{j^{\prime}}\left(\boldsymbol{P}_{\phi}^{(I)}\right. & =\max _{1 \leqq k \leqq n} \prod_{i=1}^{n} p_{i}^{y i^{i}(I)} \bar{l}_{\phi}^{j^{\prime}}\left(\boldsymbol{Q}_{\phi}^{(I)}\right)=\max _{1 \leqq k \leqq m^{j}} \prod_{i=1}^{n} q_{i}^{z_{i k}^{i}+1(I)} \\
l_{\phi}^{j^{*}}\left(\boldsymbol{P}_{\phi}^{(I)}\right) & =\prod_{k=1}^{m^{j}} \prod_{i=1}^{n} p_{i}^{z_{i}^{i}+1(I)} \bar{l}_{\phi}^{j^{*}}\left(\boldsymbol{Q}_{\phi}^{(I)}\right)=\prod_{k=1}^{n} \prod_{i=1}^{n} q_{i}^{y_{i k}^{i}(I)} \\
B_{\phi}^{j}\left(\boldsymbol{P}_{\phi}^{(I)}\right) & =\max _{j \leqq k \leqq M}\left\{\max \left[l_{\phi}^{k^{\prime}}\left(\boldsymbol{P}_{\phi}^{(I)}\right), l_{\phi}^{k^{*}}\left(\boldsymbol{P}_{\phi}^{(I)}\right)\right]\right\} \\
\bar{B}_{\phi}^{j}\left(\boldsymbol{Q}_{\phi}^{(I)}\right) & =\max _{1 \leqq k \leqq j}\left\{\max \left[\bar{l}_{\phi}^{k^{\prime}}\left(\boldsymbol{Q}_{\phi}^{(I)}\right), \bar{l}_{\phi}^{k^{*}}\left(\boldsymbol{Q}_{\phi}^{(I)}\right)\right]\right\} .
\end{aligned}
$$

Then

$$
\begin{gathered}
B_{\phi}^{j}\left(\boldsymbol{P}_{\phi}^{(I)}\right) \leqq h_{\phi}^{j(I)} \leqq \inf _{t \in I}\left[1-\bar{B}_{\phi}^{j}\left(\boldsymbol{Q}_{\phi}^{(t)}\right)\right] \leqq 1-\bar{B}_{\phi}^{j}\left(\boldsymbol{Q}_{\phi}^{(I)}\right) \\
\bar{B}_{\phi}^{j}\left(\boldsymbol{Q}_{\phi}^{(I)}\right) \leqq g_{\phi}^{j(I)} \leqq \inf _{t \in I}\left[1-B_{\phi}^{j}\left(\boldsymbol{P}_{\phi}^{(t)}\right)\right] \leqq 1-B_{\phi}^{j}\left(\boldsymbol{P}_{\phi}^{(I)}\right) . \\
\prod_{i=1}^{n} a_{i} \stackrel{\text { def }}{=} 1-\prod_{i=1}^{n}\left(1-a_{i}\right) .
\end{gathered}
$$

The poorer upper bounds to the right are those of Funnemark and Natvig (1985).

Proof. We give the proof for the improved upper bound of $h_{\phi}^{j(I)}$; the corresponding proof for $g_{\phi}^{j(I)}$ is entirely similar. By applying Corollary 3.7 of Funnemark and Natvig (1985) for each fixed $t \in I$, we get

$$
h_{\phi}^{j(I)} \leqq \inf _{t \in I} h_{\phi}^{j(t)} \leqq \inf _{t \in I}\left[1-\bar{B}_{\phi}^{j}\left(Q_{\phi}^{(t)}\right)\right]
$$

Noting that for each fixed $t \in I$

$$
\bar{B}_{\phi}^{j}\left(\boldsymbol{Q}_{\phi}^{(t)}\right) \geqq \bar{B}_{\phi}^{j}\left(\boldsymbol{Q}_{\phi}^{(I)}\right),
$$

the proof is completed.

It must be admitted that these improved upper bounds can be poor, as the reader will realize from the proof above. A case study where these bounds are involved is given in Natvig et al. (1986). The improved upper bounds for the unavailabilities are here much better than the original ones. This is not true for the corresponding upper bounds for the availabilities.

\section{Acknowledgement}

We are thankful to Renato Monteiro, a student of Professor Richard Barlow, for a thought-provoking comment leading to this paper. 


\section{References}

FunNEMARK, E. AND NATVIG, B. (1985) Bounds for the availabilities in a fixed time interval for multistate monotone systems. Adv. Appl. Prob. 17, 638-655.

NATVIG, B. (1980) Improved bounds for the availability and unavailability in a fixed time interval for systems of maintained, interdependent components. Adv. Appl. Prob. 12, 200-221.

Natvig, B., Sørmo, S., Holden, A. T. and HøGÅsen, G. (1986) Multistate reliability theory-a case study. Adv. Appl. Prob. 18 (to appear). 